CASE REPORT OPEN

\title{
Trastuzumab and pertuzumab in circulating tumor DNA ERBB2-amplified HER2-positive refractory cholangiocarcinoma
}

\author{
Bhavya Yarlagadda (iD), Vaishnavi Kamatham ${ }^{1}$, Ashton Ritter ${ }^{1}$, Faisal Shahjehan ${ }^{1}$ and Pashtoon M. Kasi ${ }^{2}$
}

Cholangiocarcinoma is a heterogeneous and target-rich disease with differences in actionable targets. Intrahepatic and extrahepatic types of cholangiocarcinoma differ significantly in clinical presentation and underlying genetic aberrations. Research has shown that extrahepatic cholangiocarcinoma is more likely to be associated with ERBB2 (HER2) genetic aberrations. Various anti-HER2 clinical trials, case reports and other molecular studies show that HER2 is a real target in cholangiocarcinoma; however, anti-HER2 agents are still not approved for routine administration. Here, we show in a metastatic cholangiocarcinoma with ERBB2 amplification identified on liquid biopsy (circulating tumor DNA (ctDNA) testing), a dramatic response to now over 12 months of dual-anti-HER2 therapy. We also summarize the current literature on anti-HER2 therapy for cholangiocarcinoma. This would likely become another treatment option for this target-rich disease.

npj Precision Oncology (2019)3:19; https://doi.org/10.1038/s41698-019-0091-4

\section{INTRODUCTION}

Cholangiocarcinoma (CCA) is a lethal tumor arising from the epithelium of the bile ducts that most often presents at an advanced stage. A total of $10-20 \%$ of primary hepatic malignancies are CCA, which is the second most common hepatobiliary malignancy. ${ }^{1}$ Risk factors for development of CCA include cigarette smoking, heavy alcohol use, primary sclerosing cholangitis, viral hepatitis $B$ and $C$, and genetic diseases such as Lynch syndrome. ${ }^{2}$

CCA, including gallbladder cancer, is a heterogeneous group of diseases. Multiple aberrations can be seen, including ERBB2, microsatellite instability high (MSI-H), IDH1, BRAF, FGFR fusion, BRCA/DNA-repair related, MET amplification, NTRK fusion, TP53, KRAS, ARID1A, MCL1, PBRM1, SMAD4, FBXW7, and CDKN2A. ${ }^{3-10}$ As in most cancers, these aberrations are not actionable; however, cholangiocarcinoma is a very "target rich" disease; ERBB2, MSI- $H_{\text {, }}$ IDH1, BRAF, FGFR fusion, MET amplification, and NTRK fusion ${ }^{5,6,8,9,11}$ may be susceptible to approved or off-label or presence of drugs treatments through clinical trials that are showing promise. Tumor-agnostic FDA-approved immunotherapy for MSI-H tumors and larotrectinib for NTRK-fusion tumors are showing promise. ${ }^{9,12}$ A number of published cases and open clinical trials with early results have demonstrated activity in IDH1, BRAF-mutant, METamplified, ERBB2-amplified, and FGFR-fusion tumors. $6,7,13,14$

The ERBB2 gene encodes for the protein HER2 or HER2/neu, which is a tyrosine kinase family receptor. Breast, stomach and esophageal cancers have well-established associations with ERBB2 genetic aberrations; and are approved for anti-HER2. ${ }^{15,16}$ However, reports have also shown the finding of HER2 aberrations in CCA and urinary bladder cancers. ${ }^{17}$ These are interestingly seen more with extrahepatic cholangiocarcinomas and gallbladder cancers as opposed to intrahepatic cholangiocarcinomas.
We present a 71-year-old female diagnosed with metastatic CCA with ERBB2 (HER2) 3+ amplification determined by circulating tumor DNA (ctDNA) testing ("liquid biopsy") and confirmed by tissue-based testing. She was treated with dual HER2-directed therapy (trastuzumab/pertuzumab), and responded very well with regression of tumor on imaging as well as normalization of liver function tests and tumor marker levels including improvement in her overall clinical status. Serial ctDNA testing (Table 1) alongside standard of care imaging continues to show ongoing durable control $>12$ months into therapy. Other case reports and studies highlighting the association of HER2 and CCA are also presented. The patient provided written informed consent to report this case.

\section{RESULTS}

Case presentation

A 71-year-old female presented in December 2017 after diagnosis of metastatic CCA. Ultrasound demonstrated innumerable liver lesions, which on confirmed on follow-up computed tomography and magnetic resonance imaging showing multiple liver lesions consistent with CCA with intrahepatic metastases. The patient was also noted to have metastatic periportal and aortocaval adenopathy.

The patient was not a candidate for surgical intervention due to bilobar disease with innumerable liver lesions. Platinum-based chemotherapy was recommended. She was started on a combination carboplatin and gemcitabine (not cisplatin due to age and sensorineural hearing loss). A baseline ctDNA test was obtained as part of the standard of care at our institution for patients with gastrointestinal malignancies, and in particular cholangiocarcinoma, given the target-rich nature of the disease. Testing is performed through commercially available platforms.

\footnotetext{
${ }^{1}$ Division of Oncology, CORPS Program, Department of Internal Medicine, Mayo Clinic, Jacksonville, FL, USA and ${ }^{2}$ Division of Hematology, Oncology and Blood \& Bone Marrow Transplantation, Department of Internal Medicine, University of lowa, lowa City, IA, USA Correspondence: Pashtoon M. Kasi (pashtoon-kasi@uiowa.edu)
}

Received: 22 March 2019 Accepted: 26 July 2019

Published online: 19 August 2019 
Table 1. Serial circulating tumor DNA evaluation in our patient

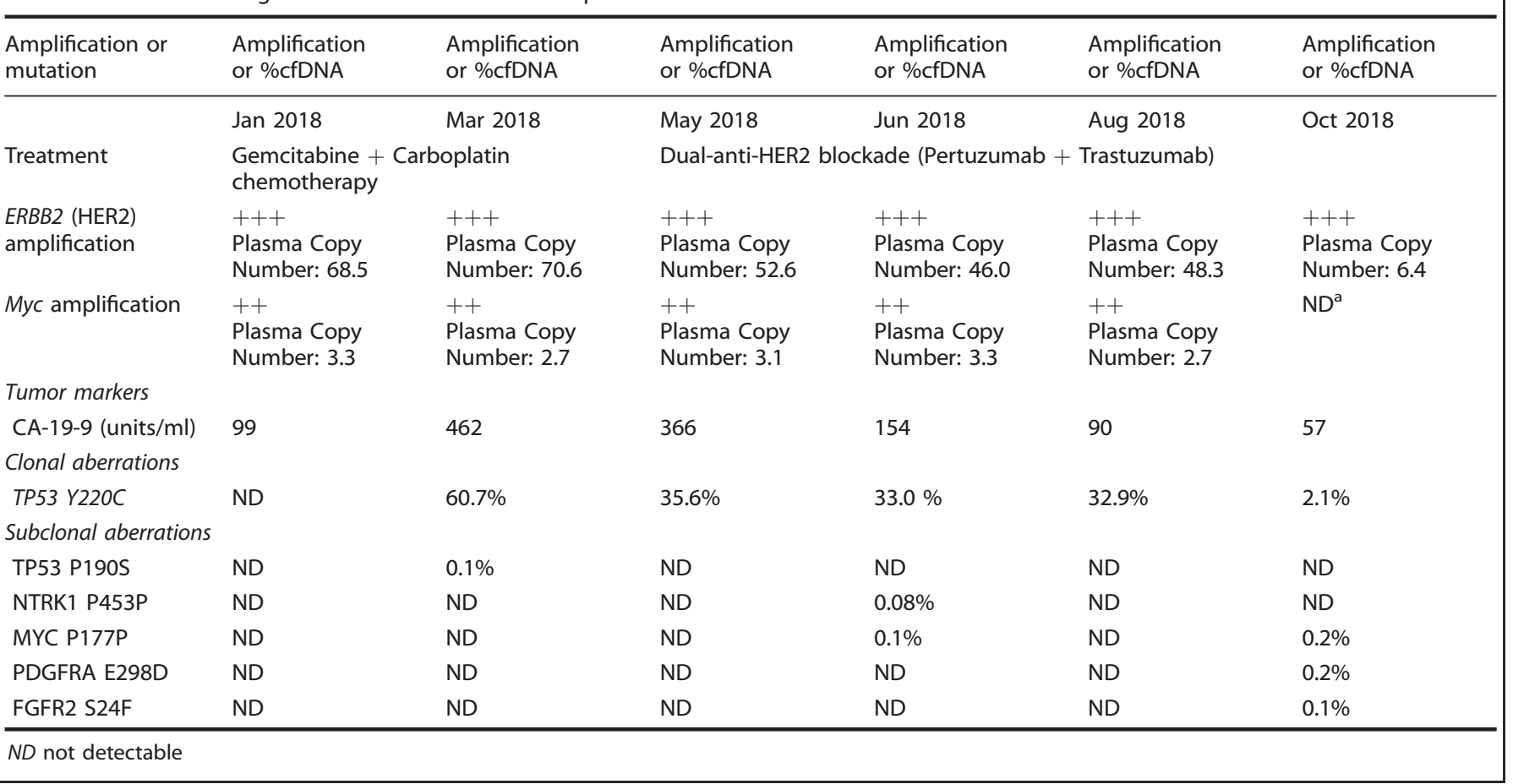



Fig. 1 Tumor with intense HER2/Neu 3+ positivity on immunohistochemistry (scale $50 \mu \mathrm{m}$ )

Guardant360 testing showed ERBB2 (HER2) amplification 3+ and was confirmed through tumor tissue-based immunohistochemistry as well as genetic testing through the commercially available platform by Tempus confirming this (Fig. 1). Given the liverpredominant nature of the disease, upfront Y90-radioembolization was also planned. However, within 2 months, the patient had rapid progression of disease with rising tumor markers, rising ctDNA levels, derangement in liver function tests and decline in clinical condition.

We initially considered the patient's eligibility for the MyPathway Study which has an arm for dual-anti-HER2 therapy. ${ }^{18}$ However, due to her rapid decline was deemed ineligible. Best supportive/palliative care vs. off-label anti-HER2 therapy was discussed. Given the patient's excellent overall baseline performance status, we began off-label treatment with anti-HER2 pertuzumab/trastuzumab combination therapy.

Immediate and rapid improvement of tumor markers was noted. After just one treatment, the patient's liver function tests improved; most notably, the dominant TP53 mutation reduced from 60.7 to $2.1 \%$ (Table 1). Clinical variables continued to improve rapidly through and continues to do so now over a year into treatment. Scans also showed excellent ongoing durable response (Fig. 2a, b).

\section{DISCUSSION}

CCA is classified into intrahepatic and extrahepatic types based on anatomic location. These are clinically different from each other in terms of presentation, and more importantly the type and frequency of genetic aberrations. Generally, intrahepatic CCA is associated with IDH1, FGFR fusion and BRCA/DNA-repair gene alterations, while extrahepatic CCA is more frequently associated with ERBB2 genetic aberrations. ${ }^{19}$ A number of these aberrations are potentially actionable in terms of FDA-approved therapies and/or availability of clinical trials or off-label therapies. For a rare disease like CAA, even the latter are pertinent. There are several well-established risk factors related to the development of CCA. However, it has been reported that only $10 \%$ of intrahepatic CCA is associated with these risk factors and the remaining $90 \%$ are sporadic. $^{20}$

What is unique about our case is that the HER2 aberration was detected on circulating tumor DNA testing ("liquid biopsy"). This was later confirmed on tissue-based testing (Fig. 1). This would not have been known otherwise. Furthermore, for a patient with refractory cholangiocarcinoma that progresses on frontline therapy, prognosis in such situations is in the order of weeks-months. The fact that patient continues to have durable control 12 months and beyond is very encouraging and unprecedented. Progression-free survival of second-line chemotherapeutic regimens is in the order of 4 months on average.

Several studies have described the association of CCA subgroups with various aberrations (Table 2). Churi et al. examined 75 CCA patients for tumoral genetic differences. They identified TP53, KRAS, ARID1A, IDH1, MCL1, and PBRM1 mutations in 35\%, $24 \%, 20 \%, 18 \%, 16 \%$, and $11 \%$ of intrahepatic CCA patients, respectively. TP53, KRAS, ERBB2, SMAD4, FBXW7, and CDKN2A were 


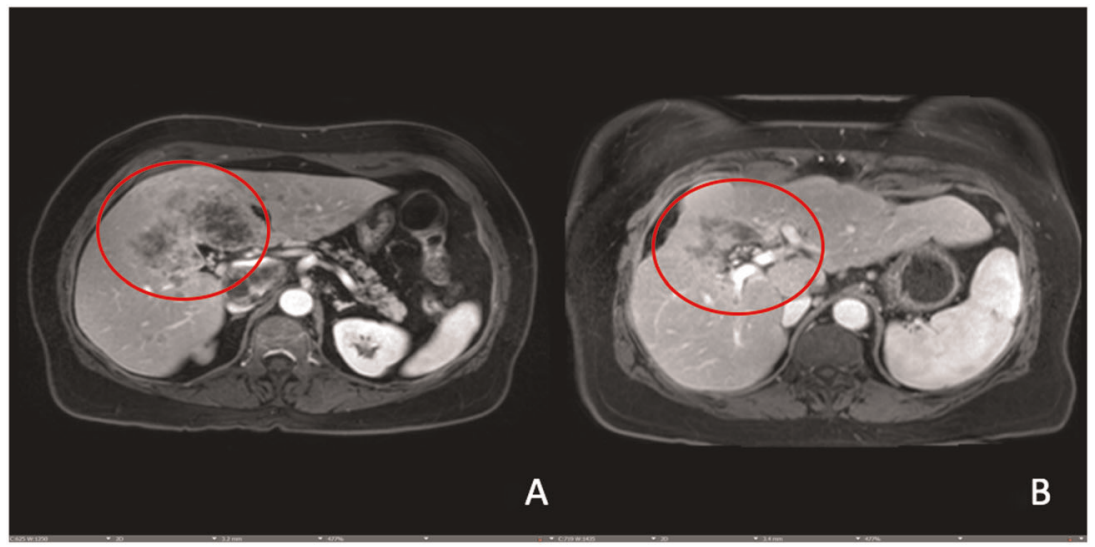

Fig. 2 MRI pre- and post-treatment with decreased size and enhancement of lesions in liver

found in $45 \%, 40 \%, 25 \%, 25 \%, 15 \%$, and $15 \%$ of extrahepatic CCA patients, respectively. ${ }^{19}$ The finding of a higher proportion of ERBB2 mutations in extrahepatic CCA and gallbladder cancer suggests that these patients are more likely to carry it as opposed to intrahepatic CCA. Yoshikawa et al. measured HER2/neu expression in 236 cases of CCA by immunohistochemistry. They reported a $0.9 \%$ and $8.5 \%$ positive HER2/neu expression rate in intrahepatic and extrahepatic CCAs, respectively. ${ }^{21}$ In a study by Lee et al., HER2 and HER3 overexpression was observed in $6 \%$ and $39 \%$, respectively, in patients with extrahepatic CCA, with HER3 overexpression associated with worse survival. ${ }^{22} \mathrm{~A}$ systemic review and meta-analysis by Galdy et al. showed a stronger rate of HER2 expression in extrahepatic CCA $(\sim 20 \%)$ vs. intrahepatic CCA $(<5 \%) .{ }^{23}$ Summary of the studies showing HER2 aberrations in CCA and treatment outcomes where available are presented in Table 2.

In terms of this particular assay's ability to detect ERBB2 (HER2/ neu) amplification, data were presented by the group from M.D. Anderson Cancer Center at the American Society of Clinical Oncology Conference (ASCO) 2018. ${ }^{24,25}$ Bardelli and colleagues validated the performance of the Guardant360 ctDNA assay retrospectively in a cohort of HER2/neu amplified metastatic colorectal cancer patients treated in the landmark HERACLES study (phase 2 study of lapatinib plus trastuzumab). The group investigated 48 plasma samples from 29 patients. CtDNA was identified in 47-48 samples; ERBB2 amplification was confirmed in 46 of these $47(97.9 \%)$ samples. The authors reported that a threshold of $\geq 3$ copies of ERBB2 in circulation allowed identification of $94 \%$ of fluorescence in situ hybridisation-positive patients. This threshold also correlated with clinical response. ${ }^{24,25}$ These results have been validated in other tumor types and cohorts of patients. ${ }^{25,26}$ Liquid biopsy, therefore, appears to be a reliable and valid tool to detect ERBB2 (Her2/neu), which is indeed an actionable finding across many tumor types including cholangiocarcinoma.

HER2/neu blockade has shown favorable results in cancers carrying HER2 aberrations. Javle et al. investigated the response of anti-HER2 therapy in 8 patients with HER2 mutated gallbladder cancer and showed an overall improvement in terms of disease stability $(n=3)$, partial response $(n=4)$, or complete response $(n=1)$ in their entire patient cohort. $^{27}$ In another study, the researchers studied HER2/neu expression in extrahepatic CCA patients $(n=84)$ and reported that anticancer therapy targeting HER2 receptors may be a reasonable option for these patients. ${ }^{28}$ Our patient has responded very well to anti-HER2 drugs and showed an improvement in symptoms, lab results and shrinkage in tumor size. This implicates the use of targeted therapy as a favorable option in CCA alongside other patients who received anti-HER2-based therapy (Table 2).
CCA diagnosis at an advanced stage has very poor outcomes and is often considered incurable. Valle et al. ${ }^{29}$ reported a study of metastatic CCA patients $(n=410)$, and reported median overall survival of 11.7 months and 8.1 months in a cisplatin-gemcitabine group and gemcitabine group, respectively. In the second-line setting, outcomes are extremely poor (progression-free survival, 3-4 months; overall survival, 6 months). Compared to historical reports, outcomes in patients receiving anti-HER2 therapy in $\mathrm{HER} 2 /$ neu $+\mathrm{CCA}$ is by far superior and very encouraging (Table 2)..$^{30,31}$

Our patient at one point was given a choice of hospice and was not eligible for any of the trials due to deranged liver function tests. But now on off-label dual-anti-HER2 therapy continues to be on treatment with excellent performance status without any chemotherapy for more than 12 months at present.

For rare, target-rich diseases like CCA, there is a need to consider approval and it is encouraging to see some recent tumoragnostic approvals, such as immunotherapy for MSI-H and larotrectinib for NTRK-fusion cancers. ${ }^{9,12}$ We believe our case and recent single-arm phase 2 study outcomes warrant consideration toward approval of anti-HER2 agents (trastuzumab/ pertuzumab) in patients with CCA with ERBB2 amplification who have very limited options and poor prognosis.

Furthermore, liquid biopsy testing for HER2 is not routinely performed at most institutions. Our patient's positive finding would have been missed. In target-rich diseases such as CCA, where tissue acquisition is a challenge, liquid biopsy provides a viable alternative for both diagnostic and monitoring techniques.

In conclusion, we were able to identify ERBB2 (HER2/neu) amplification on liquid biopsy in a patient with CCA and found anti-HER2 therapy as an effective treatment strategy. This will have implications for other patients with CCA in terms of identification of this target and considerations toward anti-HER2 therapy on- or off-trial.

\section{METHODS}

The patient provided written informed consent for her case to be presented. Separate IRB/FDA approval not required for off-label use of the anti-HER2 therapy or reporting of the case.

Reporting summary

Further information on research design is available in the Nature Research Reporting Summary linked to this article. 


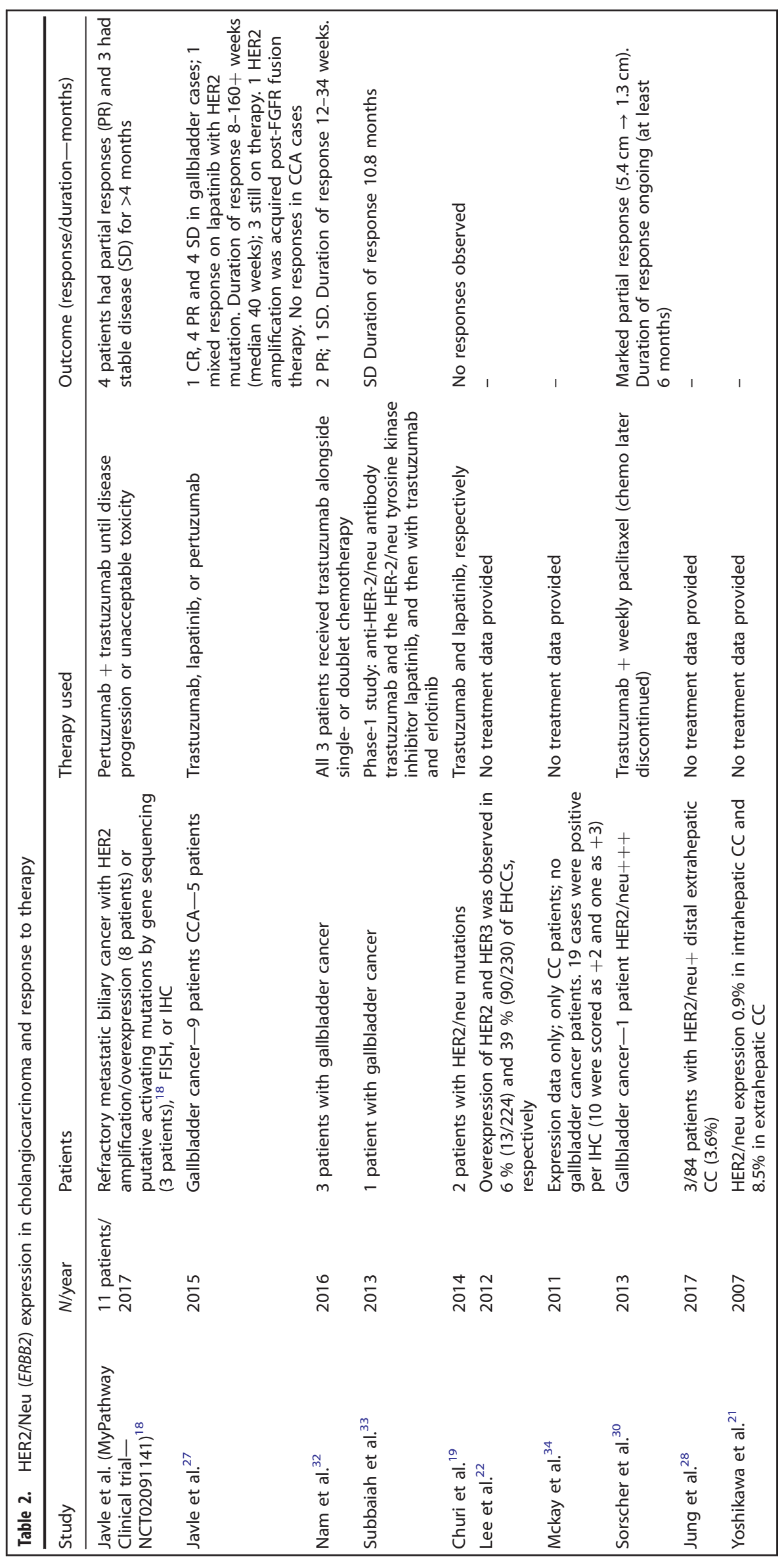




\section{DATA AVAILABILITY}

The authors declare that data supporting the findings of this study are available within the paper. Commercially available platforms were employed with results shown in the figures and tables included.

\section{AUTHOR CONTRIBUTION}

P.M.K. conceived the idea, and is the patient's primary oncologist alongside A.R. B.Y. drafted the initial draft alongside comprehensive literature review by F.S. and S.K. Additional details provided by A.R. P.M.K. revised the draft before an additional round of revisions by all authors. This was approved by all authors before submission to the journal. P.M.K., S.K., and A.R. revised the paper. All authors again approved of the final changes before resubmission.

\section{ADDITIONAL INFORMATION}

Supplementary information accompanies the paper on the npj Precision Oncology website (https://doi.org/10.1038/s41698-019-0091-4).

Competing interests: The authors declare no competing interests.

Publisher's note: Springer Nature remains neutral with regard to jurisdictional claims in published maps and institutional affiliations.

\section{REFERENCES}

1. Jusakul, A. et al. Whole-genome and epigenomic landscapes of etiologically distinct subtypes of cholangiocarcinoma. Cancer Discov. 7, 1116-1135 (2017).

2. Zhou, Z., Nie, S. D., Jiang, B., Wang, J. \& Lv, P. Risk factors for extrahepatic cholangiocarcinoma: a case-control study in China. Eur. J. Cancer Prev. https://doi. org/10.1097/cej.0000000000000468 (2018).

3. Gbolahan, O., Hashemi-Sadraei, N. \& O'Neil, B. Prolonged response to anti-PD-1 antibody therapy in chemotherapy-refractory cholangiocarcinoma with high tumor mutational burden. J. Natl Compr. Cancer Netw. 17, 644-648 (2019).

4. Winkelmann, R. et al. Microsatellite instability occurs rarely in patients with cholangiocarcinoma: a retrospective study from a german tertiary care hospital. Int. J. Mol. Sci. 19 https://doi.org/10.3390/ijms19051421 (2018).

5. Farshidfar, F. et al. Integrative genomic analysis of cholangiocarcinoma identifies distinct IDH-mutant molecular profiles. Cell Rep. 18, 2780-2794 (2017).

6. Goyal, L. et al. TAS-120 overcomes resistance to ATP-competitive FGFR inhibitors in patients with FGFR2 fusion-positive intrahepatic cholangiocarcinoma. Cancer Discov. https://doi.org/10.1158/2159-8290.CD-19-0182 (2019).

7. Mazzaferro, V. et al. Derazantinib (ARQ 087) in advanced or inoperable FGFR2 gene fusion-positive intrahepatic cholangiocarcinoma. Br. J. Cancer 120, 165-171 (2019).

8. Lavingia, V. \& Fakih, M. Impressive response to dual BRAF and MEK inhibition in patients with BRAF mutant intrahepatic cholangiocarcinoma-2 case reports and a brief review. J. Gastrointest. Oncol. 7, E98-E102 (2016).

9. Drilon, A. et al. Efficacy of larotrectinib in TRK fusion-positive cancers in adults and children. N. Engl. J. Med 378, 731-739 (2018).

10. Churi, C. R. et al. Mutation profiling in cholangiocarcinoma: prognostic and therapeutic implications. PLoS ONE 9, e115383 (2014).

11. Goyal, L. et al. A phase 2 and biomarker study of cabozantinib in patients with advanced cholangiocarcinoma. Cancer 123, 1979-1988 (2017).

12. Le, D. T. et al. Mismatch repair deficiency predicts response of solid tumors to PD1 blockade. Science 357, 409-413 (2017).

13. Golub, D. et al. Mutant Isocitrate dehydrogenase inhibitors as targeted cancer therapeutics. Front. Oncol. 9, 417 (2019).

14. Meric-Bernstam, F. et al. Pertuzumab plus trastuzumab for HER2-amplified metastatic colorectal cancer (MyPathway): an updated report from a multicentre, open-label, phase 2a, multiple basket study. Lancet Oncol. 20, 518-530 (2019).

15. Loibl, S. \& Gianni, L. HER2-positive breast cancer. Lancet 389, 2415-2429 (2017).
16. Kumler, I., Tuxen, M. K. \& Nielsen, D. L. A systematic review of dual targeting in HER2-positive breast cancer. Cancer Treat. Rev. 40, 259-270 (2014).

17. Latif, Z. et al. HER2/neu overexpression in the development of muscle-invasive transitional cell carcinoma of the bladder. Br. J. Cancer 89, 1305-1309 (2003).

18. Javle, M. M. et al. Herbert Hurwitz Pertuzumab + trastuzumab for HER2-positive metastatic biliary cancer: preliminary data from MyPathway. J. Clin. Oncol. 35, 402 (2017).

19. Churi, C. R. et al. Mutation profiling in cholangiocarcinoma: prognostic and therapeutic implications. Plos ONE 9, e115383 (2014).

20. Ben-Menachem, T. Risk factors for cholangiocarcinoma. Eur. J. Gastroenterol. Hepatol. 19, 615-617 (2007).

21. Yoshikawa, D. et al. Clinicopathological and prognostic significance of EGFR VEGF, and HER2 expression in cholangiocarcinoma. Br. J. Cancer 98, 418-425 (2008)

22. Lee, H. J., Chung, J.-Y., Hewitt, S. M., Yu, E. \& Hong, S.-M. HER3 overexpression is a prognostic indicator of extrahepatic cholangiocarcinoma. Virchows Arch. 461, 521-530 (2012).

23. Galdy, S. et al. HER2/HER3 pathway in biliary tract malignancies; systematic review and meta-analysis: a potential therapeutic target? Cancer Metastas. Rev. 36, 141-157 (2017)

24. Bardelli, A. et al. Plasma HER2 (ERBB2) copy number to predict response to HER2targeted therapy in metastatic colorectal cancer. J. Clin. Oncol. 36, 3506-3506, https://doi.org/10.1200/JCO.2018.36.15_suppl.3506 (2018).

25. Siravegna, G. et al. Plasma HER2 (ERBB2) copy number predicts response to HER2targeted therapy in metastatic colorectal cancer. Clin. Cancer Res. 25, 3046-3053 (2019).

26. Lee, J. et al. Detection of ERBB2 (HER2) gene amplification events in cell-free DNA and response to anti-HER2 agents in a large asian cancer patient cohort. Front. Oncol. 9, 212 (2019).

27. Javle, M. et al. HER2/neu-directed therapy for biliary tract cancer. J. Hematol. Oncol. 8, 58 (2015).

28. Jung, M. J. et al. Gene copy number variation and protein overexpression of EGFR and HER2 in distal extrahepatic cholangiocarcinoma. Pathology 49, 582-588 (2017)

29. Valle, J. et al. Cisplatin plus gemcitabine versus gemcitabine for biliary tract cancer. New Engl. J. Med. 362, 1273-1281 (2010).

30. Sorscher, S. Marked radiographic response of a HER-2-overexpressing biliary cancer to trastuzumab. Cancer Manag. Res. 9, 1-3 (2013).

31. Fornaro, L. et al. Second-line chemotherapy in advanced biliary cancer progressed to first-line platinum-gemcitabine combination: a multicenter survey and pooled analysis with published data. J. Exp. Clin. Cancer Res. 34, 156 (2015).

32. Nam, A. R. et al. Therapeutic implication of HER2 in advanced biliary tract cancer. Oncotarget 7, 58007-58021 (2016).

33. Subbiah, I. M. et al. Targeted therapy of advanced gallbladder cancer and cholangiocarcinoma with aggressive biology: eliciting early response signals from phase 1 trials. Oncotarget 4, 156-165 (2013).

34. McKay, S. C. et al. Array comparative genomic hybridization identifies novel potential therapeutic targets in cholangiocarcinoma. HPB 13, 309-319 (2011).

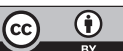

Open Access This article is licensed under a Creative Commons Attribution 4.0 International License, which permits use, sharing, adaptation, distribution and reproduction in any medium or format, as long as you give appropriate credit to the original author(s) and the source, provide a link to the Creative Commons license, and indicate if changes were made. The images or other third party material in this article are included in the article's Creative Commons license, unless indicated otherwise in a credit line to the material. If material is not included in the article's Creative Commons license and your intended use is not permitted by statutory regulation or exceeds the permitted use, you will need to obtain permission directly from the copyright holder. To view a copy of this license, visit http://creativecommons. org/licenses/by/4.0/.

(C) The Author(s) 2019 